\title{
A Vertical Asymmetry in Saccades
}

\author{
Mathias Abegg \\ Department of Ophthalmology, University of Bern, Bern, Switzerland; \\ Dario Pianezzi \\ Department of Ophthalmology, University of Bern, Bern, Switzerland;
}

Jason J.S. Barton

Departments of Medicine (Neurology), Ophthalmology and Visual Sciences, University of British Columbia, Vancouver, Canada

\begin{abstract}
Visual exploration of natural scenes imposes demands that differ between the upper and the lower visual hemifield. Yet little is known about how ocular motor performance is affected by the location of visual stimuli or the direction of a behavioural response. We compared saccadic latencies between upper and lower hemifield in a variety of conditions, including short-latency prosaccades, long-latency prosaccades, antisaccades, memory-guided saccades and saccades with increased attentional and selection demand. All saccade types except memory-guided saccades had shorter latencies when saccades were directed towards the upper field as compared to downward saccades $(\mathrm{p}<0.05)$. This upper-field reaction time advantage probably arises in ocular motor rather than visual processing. It may originate in structures involved in motor preparation rather than execution.
\end{abstract}

Keywords: antisaccade, memory-guided saccade, latency

\section{INTRODUCTION}

Visual information has different qualities in the upper and the lower hemifield. In natural environments the lower hemifield contains more information than the upper hemifield (Levine \& McAnany, 2005; Previc, 1990). This asymmetry is reflected in visual anatomy, with a larger density of ganglion cells in the upper hemiretina (Curcio \& Allen, 1990) and a greater portion of the striate cortex dedicated to processing visual information from the lower visual hemifield (Van Essen, Newsome, \& Maunsell, 1984). In turn, there are parallel vertical hemifield differences in a variety of human visual processes, such as contrast sensitivity, motion perception, visual search and letter recognition (Altpeter, Mackeben, \& TrauzettelKlosinski, 2000; Breitmeyer, Julesz, \& Kropfl, 1975; Cameron, Tai, \& Carrasco, 2002; Carrasco, Giordano, \& McElree, 2004; Danckert \& Goodale, 2001; Lakha \& Humphreys, 2005; Mackeben, 1999; Rezec \& Dobkins, 2004; Rubin, Nakayama, \& Shapley, 1996).
Asymmetries may also be present in visual attention, though here there are some discrepancies. Some have argued that transient attention leads to a larger boost of performance for stimuli in the upper hemifield (Kristjansson \& Sigurdardottir, 2008). On the other hand, others report that the spatial resolution of attention is greater in the lower hemifield $(\mathrm{He}, \mathrm{Cavanagh}$, \& Intriligator, 1996).

Given the close link between visual perception, attention and eye movements, it is plausible that there may also be vertical hemifield asymmetries in ocular motor function. Previous research in monkeys found that the latencies of prosaccades directed to the upper hemifield were shorter than for those directed to the lower hemifield (Zhou \& King, 2002; Bell, Everling, \& Munoz, 2000). This observation was also made in human prosaccades (Dafoe, Armstrong, \& Munoz, 2007; Goldring \& Fischer, 1997; Honda \& Findlay, 1992). Shorter latencies for upward antisaccades were found by some (Goldring \& Fischer, 1997) but not by others (Dafoe et al., 2007). 
In the current study we performed a comprehensive evaluation of saccades executed under a range of perceptual and ocular motor demands. We investigated whether the saccadic system is specifically adapted or tuned to the difference of the demands of the upper and the lower visual hemifield. For this purpose we compared saccadic latencies of saccades to upper and lower targets in a variety of paradigms including prosaccades, antisaccades, memory-guided saccades, saccades toward complex stimuli and saccades toward dimly visible stimuli.

\section{GENERAL METHODS}

\section{Subjects}

The study involved 35 subjects, 17 males and $18 \mathrm{fe}$ males. All participants had normal or corrected-to-normal vision. 7 subjects participated in more than one experiment. The median age of all participants was 33 years (range $20-46$ ), and the median age was similar across all experiments (experiment 1: 33, experiment 2: 34, experiment $3 ; 33$, experiment $4: 33$, experiment $5: 24)$. The protocol was approved by the institutional review boards of Vancouver General Hospital and the University of British Columbia, and all subjects gave informed consent in accordance with the Code of Ethics of the World Medical Association (Declaration of Helsinki).

\section{Apparatus}

For experiment $1-4$, subjects sat $60 \mathrm{~cm}$ away from a 19" CRT-monitor (ViewSonic G90fb) that had dimensions of 1024 pixels by 768 pixels and a refresh rate of $100 \mathrm{~Hz}$. In experiment 5 we used a smaller monitor (17') with a lower refresh rate $(85 \mathrm{~Hz})$ at $40 \mathrm{~cm}$ viewing distance, which allows easier estimates of the impact of the refresh rate on saccadic latency. Head position was stabilized by forehead and chin rests. Experiments were carried out in bright room-light conditions, which were kept constant across all experiments. Stimuli were created using Experiment Builder 1.5.1 (SR Research, Mississauga, Canada) and eye movements recorded at $1000 \mathrm{~Hz}$ using the SR Research EyeLink 1000 system. All subjects were calibrated with a nine-point array and calibration was judged successful if all nine calibration points had an accuracy of at least $1^{\circ}$.

\section{Stimuli}

Each trial started with the presentation of a fixation cross $\left(1^{\circ}\right)$ in the centre of the screen. In experiment $1,2,3$ and 5 the fixation cross was black on white background. In experiment 4 the fixation cross was red. The trial started when eye position was within $1^{\circ}$ of the fixation cross for $200 \mathrm{~ms}$; if this did not occur within a 4-second period, the trial was postponed and recalibration performed. Stimuli were black discs of $1^{\circ}$ containing a central white dot, displayed on a white background unless otherwise stated. For experiment 1, 3, 4 and 5 the stimuli were located in one of 4 possible stimulus locations, at oblique angles of $45^{\circ}$ and an eccentricity of $10^{\circ}$ of visual angle from the screen centre (figure 1). Stimuli remained visible for $850 \mathrm{~ms}$ after a saccade was initiated and were then replaced by the central fixation cross to start the next trial. We instructed participants to look at the stimulus (or away from it, in the case of antisaccades) as soon as it appeared and then back at the screen centre when the fixation cross reappeared.

\section{Data analysis}

Data was analyzed using EyeLink Data Viewer 1.2.454 (SR Research, Mississauga, Canada). Saccades were detected when eye velocity exceeded $31 \%$ sec, acceleration exceeded $9100 \% \mathrm{sec}^{2}$, and position changed by more than $0.15^{\circ}$. These parameters corresponded to the default values of the eye-tracker. Only the first saccade after stimulus onset with an amplitude of more than $3^{\circ}$ was analyzed in each trial. Saccadic latency was the time from stimulus onset to saccade onset. We excluded trials with latencies less than $60 \mathrm{~ms}$, latencies longer than 800 $\mathrm{ms}$, or whose starting points were located more than $1^{\circ}$ from the central fixation cross. Trials with a directional error of more than $45^{\circ}$ were also excluded from the latency analysis. Based on these criteria, 19\% of all trials were excluded.

We used JMP 8.0.2 software (www.jmp.com) to statistically analyze the data with a general linear model. Saccadic latency was the dependent variable. Main factors are indicated in the procedure of each experiment. Contrasts for significant effects were examined with the Tukey's honestly significant different (HSD) test. In experiment 2 and 4 a paired t-test was used for statistical analysis of the error rate. 


\section{EXPERIMENT 1}

The aim of this first experiment was a simple compar-ison of the latencies of upward and downward prosaccades made in oblique directions.

\section{Procedure}

9 subjects participated. Each trials started with a central fixation cross that was followed by a blank screen (the gap). The gap's duration randomly varied between 200 and $300 \mathrm{~ms}$ in $10 \mathrm{~ms}$ increments. After the gap a target appeared in one of the four possible target locations, at $10^{\circ}$ eccentricity on the $45^{\circ}$ oblique angle of each quadrant. Over the entire experiment, the gap intervals were randomly distributed so that all four stimulus locations had the same number of a given gap interval. There were 528 trials, with a short break after the first 264 trials.

Results were analyzed with a general linear model using saccadic latency as the dependent variable and the main factor of saccade direction (upper left, upper right, lower left, lower right), with subject as a random effect.

\section{Results}

There was a main effect of saccade direction $(\mathrm{F}(3.1824)=48, \mathrm{p}<0.0001$, figure 1$)$. Tukey's HSD test showed significant differences for all contrasts except between upper left and upper right and between lower left and lower right. Thus, all contrasts between one upper and one lower field location were significant, due to shorter saccadic latencies for upper targets $(\mu=132 \pm$ 9ms) than for lower targets $(\mu=151 \pm 12 \mathrm{~ms})$.

As latency did not differ between left and right hemifields, we collapsed across horizontal locations in the following analysis. We considered whether the latency advantage for the upper hemifield resulted from a speedaccuracy trade-off, resulting in less accurate saccades to upper targets. Accuracy in a prosaccade task like this would be reflected best, not in error rate, which would be very low, but in the variability of spatial accuracy - that is, the variance of saccadic amplitude. There was a trend to lower variance for the amplitude of upward saccades $\left(\mu=1.1^{\circ} \pm 0.2\right)$ than lower saccades $\left(\mu=1.7^{\circ} \pm 0.4\right.$, $\mathrm{p}=0.052, \mathrm{t}=2.42$, paired t-test), the opposite of what would be expected from a speed-accuracy trade-off.
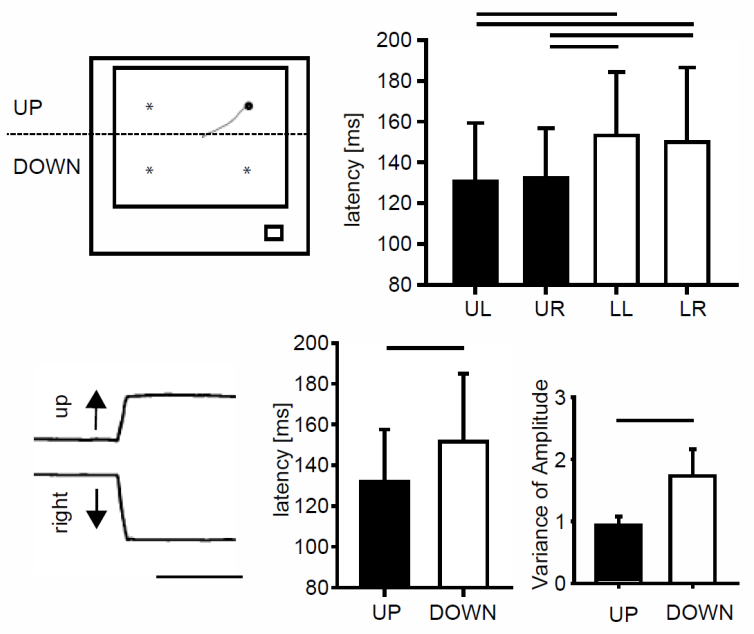

FIGURE 1. Upper left panel; experimental design shows possible location of stimuli (*) with a sample stimulus and a saccade trajectory in the upper right quadrant. Bottom left panel; example of a saccade to the target toward a target in the top right indicating rapid concurrent vertical and horizontal shift of gaze position. Scale bar corresponds to $500 \mathrm{~ms}$. Upper right panel; saccadic latency is shorter in the upper left (UL) or the upper right quadrant (UR) than the saccadic latency in the lower left (LL) or the lower right (LR) quadrant. Bottom middle panel; as no difference in saccadic latency was found between the left and the right hemifield, these were collapsed: results show shorter latencies for upward saccades to targets in the upper hemifield. Bottom right panel; Bar graph indicating that the variance of saccadic amplitude was lower for saccades aimed at targets in the upper hemifield than for saccades aimed at targets in the lower hemifield. Horizontal lines above graphs indicate significant differences between paired conditions.

\section{EXPERIMENT 2}

Experiment 1 replicated the finding in prior reports of faster prosaccades to targets in the upper hemifield (Bell et al., 2000; Honda \& Findlay, 1992). This could stem from either faster processing of stimuli in the upper field or more rapid programming of saccades with upward vectors. To discriminate between these possibilities, one can use the antisaccade task, in which subjects make saccades in the opposite direction to the stimuli. A prior study of 2 monkeys found that antisaccades directed up- 
wards in response to targets in the lower field were faster (Bell et al., 2000), suggesting that the latency difference originates in ocular motor programming. The first goal of experiment 2 was to examine antisaccade asymmetries in a larger number of human subjects to determine if the upper-field advantage was related to stimulus processing or ocular motor programming.

A second goal of experiment 2 was to evaluate whether the upper-field advantage was still found if there was a delay between stimulus onset and the cue to make the saccade, as with a memory-guided saccade. Memoryguided saccades differ from prosaccades in two ways. First, the stimulus has disappeared by the time the saccade is executed, so stimulus-related activity is reduced at the target location. Second, the prolonged stimulus-cue interval allows time for motor planning to be completed by the time the subject is instructed to make the saccade: hence any effects based on motor response preparation would be attenuated.

Thus, experiment 2 evaluated prosaccades, antisaccades and memory-guided saccades to upper and lower targets.

\section{Procedures}

We re-examined data from experiment 1 of a study in which 11 subjects had performed saccades to a range of obliquely angled target locations (Abegg, Lee, \& J.J.S., 2010). This experiment consisted of 3 blocks with 3 different saccade types: an antisaccade block, a prosaccade block with an overlap paradigm and a memory-guided saccade block with a delay of 2 seconds between the stimulus and the go signal. Stimuli and saccadic goals were randomly distributed over 24 locations around $360^{\circ}$ of polar angle - that is, at intervals of $15^{\circ}$ - with an eccentricity of $11.4^{\circ}$ of visual angle.

Each trial began with a fixation display, which showed a white fixation cross at the center of a black screen. After $750 \mathrm{~ms}$, the cross disappeared and the stimulus appeared, a white disk with a diameter of $1^{\circ}$. In the prosaccade and the antisaccade trials, the disc remained on the screen for $850 \mathrm{~ms}$ after saccade onset. The stimulus was then replaced by the fixation cross, and the next trial began. For memory-guided saccades, grey stimuli were used. They were presented for $300 \mathrm{~ms}$, followed by a grey blank screen for $300 \mathrm{~ms}$, followed by a fixation cross that disappeared after 1.7 seconds; resulting in a 2 - second memory period. Subjects were instructed to maintain fixation until the fixation light disappeared.

For the purpose of this analysis, we collapsed saccades into two groups, upward and downward saccades, and excluded trials with stimuli on the horizontal meridian. We ran a general linear model on individual trial data, with latency as the dependent variable and main factors of hemifield goal location (upwards, downwards) and saccade type (antisaccade, prosaccade and memoryguided saccade), with subject as random effect.

For the directional error rates of antisaccades, we compared the mean error rates for upper and lower responses with paired t-tests.

\section{Results}

There was also a main effect of saccade type $(\mathrm{F}(2,3175)=625, \mathrm{p}<0.0001$, figure 2$)$, with Tukey's HSD test showing that all saccade types differed from each other, with prosaccades being most rapid and antisaccades the slowest, with memory-guided saccades having latencies intermediate to these two. There was also a main effect of hemifield goal location $(F(1,3175)=14, p$ $=0.0002$ ). The key finding was a significant interaction between saccade type and hemifield goal location $(\mathrm{F}(2,3175)=6, \mathrm{p}=0.002)$. For prosaccades, we replicated the finding in experiment 1 of shorter latencies for upward $(\mu=191 \pm 10 \mathrm{~ms})$ than downward $(\mu=214 \pm 10 \mathrm{~ms})$ saccades $(F(1,3175)=20, p<0.0001)$. Upward antisaccades prompted by stimuli in the lower hemifield also had faster latencies $(\mu=323 \pm 21 \mathrm{~ms})$ than downward antisaccades promped by upper hemifield stimuli $(\mu=$ $331 \pm 22 \mathrm{~ms}, \mathrm{~F}(1,3175)=7, \mathrm{p}=0.0086)$. In contrast, there was no difference for memory-guided saccades between upward $(\mu=289 \pm 16 \mathrm{~ms})$ and downward responses $(\mu=$ $288 \pm 18 \mathrm{~ms}, \mathrm{~F}(1,3175)=0.3, \mathrm{p}=0.60)$.

Subjects made no direction errors for prosaccades and had an error rate of less than $2 \%$ for both upward and downward responses for memory-guided saccades. For antisaccades, upward responses prompted by stimuli in the lower field had a significantly lower error rate $(\mu=$ $9.98 \% \pm 3.31)$ than downward antisaccades $(\mu=17.71 \%$ $\pm 6.86)(\mathrm{p}=0.065, \mathrm{t}=2.07$, paired $\mathrm{t}$-test $)$. 


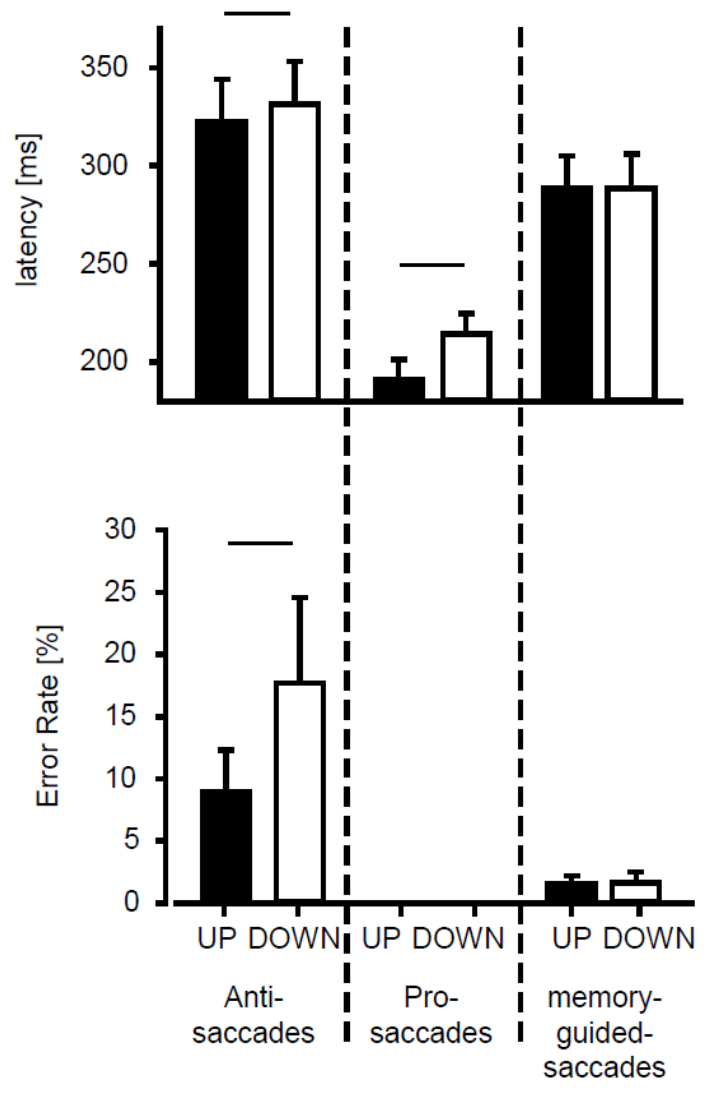

FIGURE 2. Saccadic latency and directional error rate for 3 different types of saccades: antisaccades, prosaccades and memory-guided saccades. Saccadic latency was lower for upward movements for antisaccades and prosaccades, but not memory-guided saccades. Directional error rate was lower for upward saccades as compared to downward saccades indicating that the latency advantage for upward antisaccades is not due to an accuracy trade-off. Error rate was close to zero for prosaccades and memory-guided saccades.

\section{Comment}

The prosaccade data of Experiment 2 replicated the shorter latencies of upward saccades found in Experiment 1: thus the effect is independent of differences in target locations and between overlap and gap paradigms. The finding that the antisaccades are faster when stimuli are in the lower field and responses are directed into the upper field replicates an observation made in monkeys (Bell et al., 2000), and suggests that the asymmetry may be based in ocular motor programming rather than stimulus processing. Again, the antisaccade error data indicate that this does not represent a speed-accuracy trade-off, even when accuracy is expressed as directional error rate rather than spatial accuracy. No latency difference was found for memory-guided saccade trials, in which the target is no longer visible, and which allow sufficient time for completion of motor response preparation well before the saccade needed to be made. The inference from the antisaccade and memory-guided saccade results is that the vertical asymmetry is related to motor preparation rather than either the execution of the saccade or the perceptual processing of the stimulus.

\section{EXPERIMENT 3}

The results of experiment 2 suggest that the vertical asymmetry in saccadic responses is related more to motor than sensory processing, despite the wide array of evidence we cited above of vertical asymmetries in the structure and function of human visual processing. Whether this asymmetry is related to attentional processing is of interest, given the close links between attention and ocular motor function, and the fact that a number of studies suggest vertical asymmetries in attentional processing (He et al., 1996; Kristjansson \& Sigurdardottir, 2008). So far, our prosaccade trials involved targets that place minimal demands on attention. In experiment 3 , we asked if increasing the demands on attentional selection would modify the vertical asymmetry in prosaccades. Thus we presented conjunction stimuli which require attention to select the correct target for the response (He et al., 1996).

\section{Procedures}

6 subjects participated. Each trial presented a display of 4 ' $T$ 's, one in each visual quadrant at the same location where the saccadic stimuli had been located in Experiment 1: that is, along the $45^{\circ}$ obliques at an eccentricity of $10^{\circ}$ of visual angle. The horizontal and vertical bars of the $\mathrm{T}$ both spanned $2.3^{\circ}$ of visual angle. Each of the four ' $T$ 's of a display had one of four possible orientations (upright equals $0^{\circ}, 90^{\circ}, 180^{\circ}, 270^{\circ}$ ). One of these orientations was designated the target orientation, and in each display, only one ' $\mathrm{T}$ ' had that orientation. Subjects were instructed at the beginning of the experiment that they were to make a saccade towards the ' $\mathrm{T}$ ' with that orientation. The target orientation was constant across the experiment in a given subject, but differed randomly across 
subjects. As in experiment 1, there were 528 trials, with a short break after the first 264 trials.

\section{Results}

Latencies of saccades to oriented ' $\mathrm{T}$ 's were faster for stimuli in the upper hemifield $(\mu=737 \mathrm{~ms} \pm 24$, figure 3$)$ than for stimuli in the lower hemifield $(\mu=781 \mathrm{~ms} \pm 47$, $\mathrm{F}(1,1497)=8, \mathrm{p}=0.0046)$. Although these were different subjects and formal statistical comparisons cannot be made with experiments 1 and 2, the asymmetry appears magnified compared to that seen with prosaccades to easily detected single targets (44ms versus $19 \mathrm{~ms}$ in Experiment 1 and 23ms in Experiment 2).

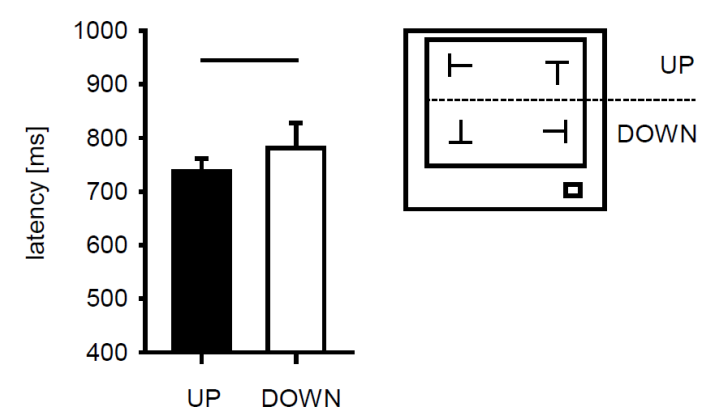

FIGURE 3. Hemifield dependence of saccadic latency in an attention-dependent task. Right panel; Illustration of experimental design with 4 simultaneously presented "T"s in different orientations. The task was to make a saccade to the $\mathrm{T}$ with a previously specified orientation. Left panel; Saccadic latency for upward saccades was shorter than that for downward saccades, the difference was similar to the one found in prosaccades, despite a significantly longer overall saccadic latency.

\section{EXPERIMENT 4}

The targets in experiment 3 were easily seen but difficult to discriminate from the foils, thus placing attentional demands for selection. Another issue is whether this asymmetry is still evident if we use stimuli that place demands upon visual detection rather than selection. In contrast to the large and bright supra-threshold targets used in Experiment 1, Experiment 4 presented nearthreshold stimuli of reduced size and contrast.

\section{Procedures}

10 subjects participated. We used small stimuli of $0.04^{\circ}$ that were adjusted to be at the detection threshold in each subject. The screen displayed four such stimuli at the four possible stimulus locations, flashing at a rate of 2 $\mathrm{Hz}$, with superimposed Gaussian noise. The threshold was determined with the method of adjustment. Using a keypress, subjects increased the grey value of the stimuli from their minimum value of 1 until they could detect the flashing stimuli. Grey values of the stimuli were then set at the maximum value of 255 and subjects decreased this until they could not see the flashing stimuli. The mean of these two values was taken as the detection threshold and used as the luminance value of the targets in the experiment for that subject. Again 512 trials were tested in each subject, with a half-way break.

The analysis of this experiment was similar as in experiment 1 , except for the error rate. We defined error rate as the proportion of all trials that were directionally incorrect. A trial was judged as directionally correct if the saccadic endpoint of the first saccade was in the same quadrant as the stimulus, and error rate was calculated as the number of incorrect trials divided by the total number of trials. The error rates between upper and lower targets were again compared with a paired t-test.

\section{Results}

There was a main effect of saccade location, with shorter latencies for saccades to upper targets $(\mu=518 \pm$ $26 \mathrm{~ms})$ than for those to the lower targets $(\mu=548 \pm$ $25 \mathrm{~ms}, \mathrm{~F}(1,2307)=19, \mathrm{p}<0.0001$, figure 4$)$. The error rate, however, was greater for upward $(\mu=29.1 \% \pm 4.0)$ than for downward saccades $(21.8 \% \pm 3.8 \%, \mathrm{p}=0.0099$, $\mathrm{t}=-3.26)$. 

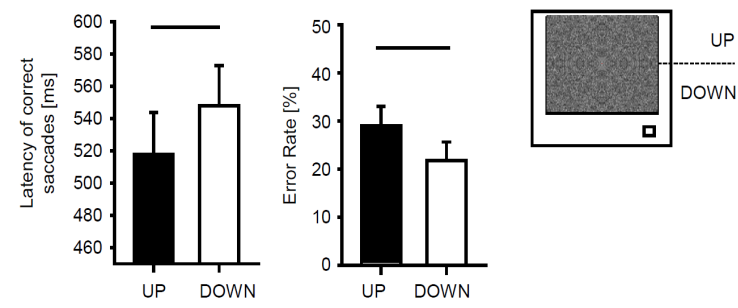

FIGURE 4. Saccadic latency to a goal with low saliency on a noisy background. Right panel shows noisy screen with a stimulus which is not visible at this magnification. Left panel; Again saccadic latency is shorter for correct upward saccades as compared to downward saccades. Middle panel; The error rate is better in the lower field, i.e. failure to make a saccades to the correct stimulus location is greater in the upper hemifield.

\section{Comment}

These result show that the upper hemifield advantage in latency is still found even if sensory processing is difficult. However, the error rates suggest a greater failure to locate the target in the upper field. While this could suggest a speed-accuracy trade-off, another interpretation is possible. Better accuracy in the lower field might suggest that detection of low-contrast targets was superior in the lower field, which would be consistent with prior studies on contrast in the vertical hemifields (Cameron et al., 2002). Hence this might indicate an interesting dissociation between a lower field advantage in sensory processing and an upper-field advantage in saccadic programming, which would be suggested by the results of Experiment 2.

\section{EXPERIMENT 5}

One possible technical artefact that could contribute to the appearance of faster responses to targets in the upper hemifield is the way CRT monitors are refreshed, which starts from the top of the screen and proceeds downwards. At a refresh rate of $100 \mathrm{~Hz}$, it would take $10 \mathrm{~ms}$ to proceed from top to bottom of the screen: given our target locations of $10^{\circ}$ visual angle at a viewing distance of $60 \mathrm{~cm}$, this would translate into a $6 \mathrm{~ms}$ difference between upper and lower targets. While the vertical asymmetries in the above experiments are larger than this, we conducted Experiment 5 to exclude this as a significant factor, by examining prosaccades with the monitor right-side up and then upside-down, in which case the screen would be refreshed from the bottom up. The average of the results of these two conditions would reveal the true behavioural effect independent of screen refresh rate.

\section{Procedures}

12 subjects participated. There were 2 blocks of prosaccades, one conducted with the monitor right-side up and the other one with the monitor upside-down. Half of the participants started with upside-down monitors. Trial design and stimuli were identical to Experiment 1. For prosaccades, participants were instructed to look at the stimulus as soon as it appeared and to look back at the center of the screen when the fixation cross reappeared. Each block contained 225 trials. For the statistical analysis we ran a general linear model with saccadic latency as dependent variable and main factors of saccadic goal direction (upper field, lower field), screen orientation (inverted or upright) and with subject as a random effect.

\section{Results}

We found significant main effects of saccade direction $(\mathrm{F}(1.3841)=151, \mathrm{p}<0.0001$, figure 5$)$, screen orientation $(\mathrm{F}(1.3841)=17, \mathrm{p}<0.0001)$ and a significant interaction $(\mathrm{F}(1.3841)=39, \mathrm{p}<0.0001)$. Tukey's HSD test confirmed a significant difference between upward and downward prosaccades with the upright screen, and also between upward and downward prosaccades with the upside-down screen. In both screen orientations, latencies of prosaccades to the upper hemifield were shorter than those to the lower hemifield. However the significant interaction was due to this difference being larger with the upright screen (upper $\mu=121 \pm 11 \mathrm{~ms}$, lower $\mu=140$ $\pm 16 \mathrm{~ms}, \mathrm{~F}(1,3841)=174, \mathrm{p}<0.0001)$ than with the inverted screen (upper $\mu=131 \pm 11 \mathrm{~ms}, \mu=135 \pm 22 \mathrm{~ms}$, $\mathrm{F}(1,3841)=14, \mathrm{p}<0.0001))$. The interaction suggests that the screen refresh strategy has a small contribution to the vertical asymmetry, but the fact that it does not reverse it means that it cannot be the sole explanation of the asymmetry. Rather, the average main effect of saccade direction suggests an $11 \mathrm{~ms}$ advantage to the upper field for prosaccades that is not accounted for by the screen refresh strategy, which would contribute a behavioural effect of about $7 \mathrm{~ms}$, similar to what we calculated above. 


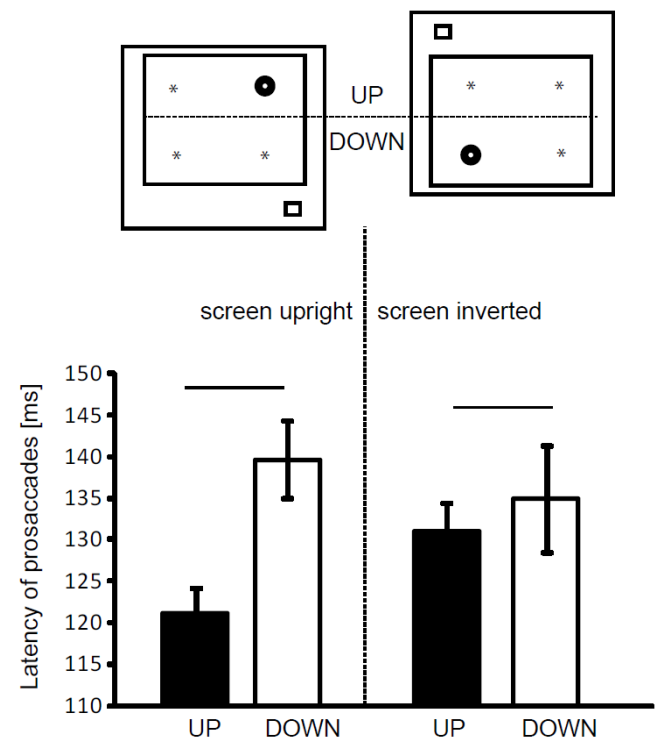

FIGURE 5. Influence of stimulus screen orientation on saccadic latency. Upper panel shows experimental design with the presentation screen in the upright position and in the inverted position. Lower panel, saccadic latency was significantly lower for upward prosaccades for both screen orientations. The latency difference between upgoing and downward saccades was reduced with the inverted screen, indicating that screen orientation indeed plays some role in calculations of saccadic latency.

\section{DISCUSSION}

Our results first confirmed the upper-field advantage in the latencies of prosaccades that has been previously reported for both monkeys and humans (Bell et al., 2000; Dafoe et al., 2007; Goldring \& Fischer, 1997; Honda \& Findlay, 1992; Zhou \& King, 2002). In experiment 2 we found an upward saccade advantage for both prosaccades and antisaccades, again consistent with previous data (Bell et al., 2000; Goldring \& Fischer, 1997), but none for memory-guided saccades. In experiments 3 and 4, we showed that the prosaccade upper-field advantage was robust. It persisted even when demands on attentional selection and visual detection were increased through the use of a conjunction search paradigm and targets with near-threshold contrast. Finally, experiment 5 showed that this upper-field advantage was not an artifact of the screen refresh strategy.
Although vertical asymmetries have been shown in a number of perceptual functions, our antisaccade study suggested that the upper-field advantage for saccades was linked to the vector of the motor response rather than the location of the sensory stimulus. This likely excludes an effect based upon known upper/lower field differences in retinal ganglion cell density (Curcio \& Allen, 1990) or striate anatomy (Van Essen et al., 1984). The conclusion that this phenomenon is motor in origin is also consistent with the results of experiment 4: while the error rate data suggested better stimulus detection in the lower visual field possibly resulting from higher retinal cell density (Curcio \& Allen, 1990), the latency data continued to show an upper-field advantage.

The only saccade type that did not show a latency dependence of the visual field were the memory-guided saccades, which required memory storage of target location during 3 seconds. In memory-guided saccades the ocular motor program may be prepared well before saccade execution. The inference from the lack of the upperfield advantage in memory-guided saccades is that the upper-field latency advantage does not originate downstream of the prepared motor response. Thus differences in burst neurons, eye muscle innervation or even muscular differences of upper and lower rectus muscles are unlikely to explain the upper-field advantage. We hypothesize that the latency difference originates in regions responsible for motor preparation. Saccade preparation involves several cortical areas including frontal eye field, parietal eye field, supplementary eye field, the dorsolateral pre-frontal cortex, as well as the superior colliculus and more (for review see (PierrotDeseilligny, Muri, Ploner, Gaymard, \& Rivaud-Pechoux, 2003)). Not all types of saccades involve all of these regions equally, though. For example, prosaccades involve a different neural network than antisaccades (Ford, Goltz, Brown, \& Everling, 2005). The fact that each saccade type we tested showed an upper-hemfield advantage suggests that a region common to all saccade types is involved. Prominent candidates may be the frontal eye field or superior colliculus, key regions in saccadic programming involved in all saccadic eye movements. At present, though, we are not aware of any data on vertical asymmetries of responses in single-cell recordings of neurons in these areas.

It is not clear if there is any functional benefit of these vertical asymmetries, or why ocular motor responses 
should show the opposite advantage to what is seen for a number of visual processes - including the saccadic localization of low contrast targets in our experiment 4 - or if these opposite advantages are related in some fashion. Our results suggest that the ocular motor programming of upward responses is more directionally and spatially accurate as well as faster, which, given the close relation of attention to ocular motor programming, may be related to the finding that transient attention boosts performance in the upper more than the lower field (Kristjansson \& Sigurdardottir, 2008). Support for an attention-mediated vertical asymmetry comes from a study by Zhou and King (2002): In two monkeys they found that the vertical asymmetry of saccadic latency is reduced if attention is moved toward a saccadic location by the use of a cue prior to saccade. From this they concluded that it may take less time to shift attention to targets in the upper visual hemifield than to targets in the lower visual hemifield, which in turn results in shorter latencies for upward saccades.

One might speculate that more efficient rapid responses in the upper hemifield come at a price, with the result that the lower hemifield possesses better spatial and attentional resolution, better detection, and improved form and motion processing. Hence it may be that all of these effects simply stem from longstanding experience that the natural environment demands greater attention and visual processing in the lower field (Levine \& McAnany, 2005; Previc, 1990).

\section{References}

Abegg, M., Lee, H., \& J.J.S., B. (2010). Systematic diagonal and vertical errors in antisaccades and memoryguided saccades. Journal of Eye Movement Research, $3(3): 5,1-10$.

Altpeter, E., Mackeben, M., \& Trauzettel-Klosinski, S. (2000). The importance of sustained attention for patients with maculopathies. Vision Research, 40(1012), 1539-1547.

Bell, A. H., Everling, S., \& Munoz, D. P. (2000). Influence of stimulus eccentricity and direction on characteristics of pro- and antisaccades in non-human primates. Journal of Neurophysiology, 84(5), 2595-2604.
Breitmeyer, B., Julesz, B., \& Kropfl, W. (1975). Dynamic random-dot stereograms reveal up-down anisotropy and left-right isotropy between cortical hemifields. Science, 187(4173), 269-270.

Cameron, E. L., Tai, J. C., \& Carrasco, M. (2002). Covert attention affects the psychometric function of contrast sensitivity. Vision Research, 42(8), 949-967.

Carrasco, M., Giordano, A. M., \& McElree, B. (2004). Temporal performance fields: visual and attentional factors. Vision Research, 44(12), 1351-1365.

Curcio, C. A., \& Allen, K. A. (1990). Topography of ganglion cells in human retina. Journal of Comparative Neurology, 300(1), 5-25.

Dafoe, J. M., Armstrong, I. T., \& Munoz, D. P. (2007). The influence of stimulus direction and eccentricity on pro- and anti-saccades in humans. Experimental Brain Research, 179(4), 563-570.

Danckert, J., \& Goodale, M. A. (2001). Superior performance for visually guided pointing in the lower visual field. Experimental Brain Research, 137(3-4), 303308 .

Ford, K. A., Goltz, H. C., Brown, M. R., \& Everling, S. (2005). Neural processes associated with antisaccade task performance investigated with event-related FMRI. Journal of Neurophysiology,, 94(1), 429-440.

Goldring, J., \& Fischer, B. (1997). Reaction times of vertical prosaccades and antisaccades in gap and overlap tasks. Experimental Brain Research, 113(1), 88-103.

He, S., Cavanagh, P., \& Intriligator, J. (1996). Attentional resolution and the locus of visual awareness. Nature, 383(6598), 334-337.

Honda, H., \& Findlay, J. M. (1992). Saccades to targets in three-dimensional space: dependence of saccadic latency on target location. Perception and Psychophysics, 52(2), 167-174.

Kristjansson, A., \& Sigurdardottir, H. M. (2008). On the benefits of transient attention across the visual field. Perception, 37(5), 747-764.

Lakha, L., \& Humphreys, G. (2005). Lower visual field advantage for motion segmentation during high competition for selection. Spatial Vision, 18(4), 447-460.

Levine, M. W., \& McAnany, J. J. (2005). The relative capabilities of the upper and lower visual hemifields. Vision Research, 45(21), 2820-2830. 
Mackeben, M. (1999). Sustained focal attention and peripheral letter recognition. Spatial Vision, 12(1), 5172.

Pierrot-Deseilligny, C., Muri, R. M., Ploner, C. J., Gaymard, B., \& Rivaud-Pechoux, S. (2003). Cortical control of ocular saccades in humans: a model for motricity. Progress in Brain Research, 142, 3-17.

Previc, F. H. (1990). Functional specialisation in the lower and upper visual fields in humans: its ecological implications. Behavior and Brain Sciences, 13, 519542.

Rezec, A. A., \& Dobkins, K. R. (2004). Attentional weighting: a possible account of visual field asymmetries in visual search? Spatial Vision, 17(4-5), 269293.
Rubin, N., Nakayama, K., \& Shapley, R. (1996). Enhanced perception of illusory contours in the lower versus upper visual hemifields. Science, 271(5249), 651-653.

Van Essen, D. C., Newsome, W. T., \& Maunsell, J. H. (1984). The visual field representation in striate cortex of the macaque monkey: asymmetries, anisotropies, and individual variability. Vision Research, 24(5), 429-448.

Zhou, W., \& King, W. M. (2002). Attentional sensitivity and asymmetries of vertical saccade generation in monkey. Vision Research, 42(6), 771-779. 